\title{
THE ECOLEAD COLLABORATIVE BUSINESS INFRASTRUCTURE FOR NETWORKED ORGANIZATIONS
}

\author{
Ricardo J. Rabelo ${ }^{1}$; Sergio Gusmeroli ${ }^{2}$ \\ ${ }^{1}$ Federal University of Santa Catarina, BRAZIL, rabelo@das.ufsc.br \\ ${ }^{2}$ TXT e-solutions, ITALY, sergio.gusmeroli@txt.it
}

This paper points out the need of advanced collaborative business ICT infrastructures (CBI) for CNOs, the requirements for the development of CBIs, and the technologies and trends considering $\mathrm{CNO}$ issues. The $\mathrm{CBI}$ devised in the ECOLEAD Project is also presented, showing how most of these requirements and emergent ICTs have been incorporated in it. This CBI called $I C T-I-$ is a distributed, open and security-embedded infrastructure, and it relies on the service oriented architecture paradigm. Its services are to be used under the on demand and pay-per-use models. The assessment of ICT-I, some conclusions and challenges are presented in the end.

\section{INTRODUCTION}

The adoption of the Collaborative Networks Organizations (CNO) paradigm by organizations imposes deep changes on the way they operate, both at intra and interenterprise levels. In resume of what several authors have presented recently (e.g. Camarinha-Matos et al., 2005), it could be said that these changes are related to three essential pre-conditions necessary to support the realization of the CNO concept. The first one is that working as a CNO requires collaboration among partners at a level far beyond sending e-mail messages. The second one is trust, considering that partners shall rely on each other. The third one is that all the transactions within the $\mathrm{CNO}$ should be digital, made via computer networks.

One of the several issues that have to be tackled to support this scenario is related to the required supporting collaborative business infrastructures (CBI). In essence, a CBI for CNOs should enable networked organizations to agilely define and set up relations with other organizations seamlessly, and to be adaptive according to the business environment conditions and current organizations' autonomy levels (Camarinha-Matos and Afsarmanesh, 2004).

Despite the complexity the development of such kind of CBI represents, the fact is that current software solutions neither attend these requirements at all nor offer an integrated support to several CNO-related business processes.

This paper aims to present the CBI implemented in the ECOLEAD project, which is called Plug and Play Horizontal ICT Infrastructure (ICT-I). ICT-I is a $\mathrm{SOA} /$ web-services-based open platform for CNOs, comprising a set of integrated tools, and that was devised to be easy-to-use and also affordable to SMEs.

This paper is organized as follows. Chapter 1 highlights the need of CBIs for CNOS. Chapter 2 presents general requirements for that as well as some obstacles 
towards the development of advanced CBIs. Chapter 3 presents the ICT-I. Chapter 4 describes the implemented services of ICT-I. Chapter 5 makes a general analysis of ICT-I, pointing out its features, innovative aspects and limitations.

\section{REQUIREMENTS FOR A CBI TO CNO}

CNOs have a different sort of business processes that is not handled by B2B and EIA solutions. Actually, CNO processes complement the processes managed by such solutions. CNO processes use to be interactive/user-centric, asynchronous and not necessarily well structured or defined a priori. Their main focus is on flexibility and adaptability, rather than on execution efficiency. Figure 1 lists just some CNOrelated processes (at application level) involved in the life cycle of a CNO of type Virtual Organization (VO), which should then be supported by CBIs to CNOs.

\begin{tabular}{|l|}
\hline VO Creation \\
- Business Opportunity characterization \\
- Selection of performance indicators \\
- Partner Search \\
- Partner Selection \\
- Negotiation \& Risk Analysis \\
- E-Contracting \\
- VO Planning
\end{tabular}

VO Operation \& Evolution
- VO Launching
- VO operational governance
- Dynamic VO management
- VO performance measurement
- Business Process supervision
- Collaborative decision-making
- VO simulation

\section{Vo Dissolution}

- VO inheritance

- Partners assessment

- IPR Management

- Checking contract

- Security access cancellation

- Legal issues

- VBE members rewarding

VO lifecycle

Figure 1 - Example of CNO-related collaborative processes

As it can be realized, a CBI for CNOs is much more than a platform for CSCW. Ideally, a CBI for CNOs should provide functionalities for enabling ${ }^{1}$ : people to collaborate and negotiate; systems / services to execute and adapt; knowledge and information (at all levels) to be exchanged and retrieved; computing and human resources to be discovered and shared; processes to be interconnected and synchronized. Managing this with high efficiency, cleverness and transparency is one of the toughest challenges to be faced by advanced CBIs.

From the technological point of view, the consideration of emerging business models and ICTs in the design of advanced CBIs is of paramount importance as they represent elements that will increasingly be incorporated into the next generation of eco-systems, which are implemented in a diversity of platforms, equipments and ubiquitous devices. Figure 2 resumes an interpretation on the NESSI roadmap ${ }^{16}$, compared to the general nowadays' reality. This aims to characterize past and future values in terms of CBIs for CNOs, where the aspects of collaboration flexibility and human intervention start to be as important as process efficiency and full automation. This reflects a scenario where compound and autonomous modules of software - deployed in several repositories, and seen services and as utilities (SaaS + U) - can act with cleverness and flexibility to solve problems and to adapt themselves to changes in the business environment, having the human being as the centre of actions and decisions. 


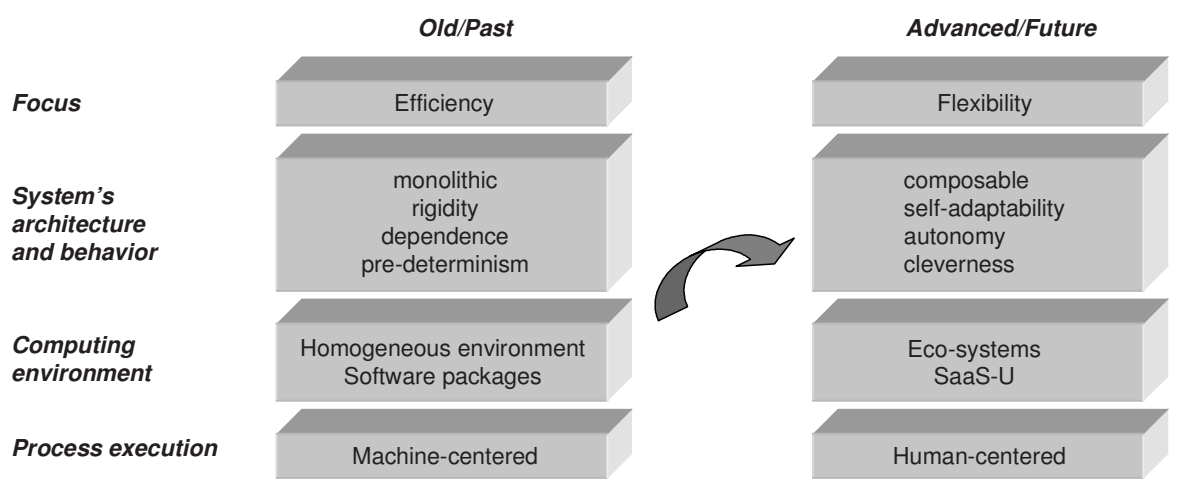

Figure 2 - Shift on the focus of collaborative business ICT infrastructures

\section{DEVELOPED CBI: THE ECOLEAD ICT-I}

The conception of ICT-I tried to follow the vision presented in Figure 2. Nevertheless, developing a completely transparent, fully interoperable and totally reliable $\mathrm{CBI}$ to cope with all $\mathrm{CNO}$ requirements is not possible considering the limitations of current ICTs and a good number of related research problems. Moreover, $\mathrm{CNO}$ is an emergent area and many related issues are still gaining ground, which means the existence of several open questions. In this sense, the strategy adopted in ICT-I was to design a generic/reference architecture and flexible framework in way it can evolve as long as newer CNO models and ICTs are introduced and open questions are solved.

The essential idea of ECOLEAD ICT-I is based on the vision of a plug \& play infrastructure. This means that any $\mathrm{CNO}$ member is provided with adequate tools to be "plugged" into the ICT-I / CNO community and to "play" (i.e. to collaborate with other organizations) in a secure, on-demand and pay-per-use ways. In direction to cope with this need, ICT-I has been fully developed based on open and platformindependent specifications and ICT standards.

Regarding its features and potentialities, ICT-I applies the Service Oriented Architecture (SOA) approach, and web-services (WS) is the core technology that has been used to implement ICT-I. Following SOA principles, and aiming at supporting the desired flexibility, ICT-I has been designed as a distributed open bus composed of many (distributed) services which are accessed on demand to support CNO members in doing businesses and collaborating.

It is important to point out that ICT-I is not a framework for SOA-based developments (like IBM WebSphere ${ }^{2}$, SAP NetWeaver ${ }^{3}$ or Oracle Fusion ${ }^{4}$ ), nor an integrated CSCW/Groupware package (like Lotus $^{5}$ or PHPCollab ${ }^{6}$ ), nor another B2B middleware (like Microsoft BizTalk ${ }^{7}$ ), and nor a proprietary services-based platform (like $\left.D B E^{16}\right)$. Actually, any of these SOA frameworks could be used to develop (web-)services (WS) for CNOs. In ECOLEAD, all the developed services have utilized $A X I S^{8}$ framework, which is robust, open-source, non-proprietary and compliant to the $\mathrm{W} 3 \mathrm{C}$ recommendations (www.w3c.org). 


\subsection{ICT-I Scope}

ICT-I acts as a CNO collaborative bus, allowing different and distributed organizations to interact with each other. ICT-I functionalities are modeled as services (see next sections) and high-level applications (ICT-I clients) can have access to them via web portals and/or via invoking ICT-I services directly. ICT-I can then be used as the ICT "glue" to link all those elements, also including CNO members' legacy systems. Figure 3 illustrates this scenario and the ICT-I scope. Services (ICT-I and services-based applications) are registered, deployed and maintained in distributed repositories, which are logically joined in a common area called Services Federation (see section 3.4). This distribution is, however, totally transparent to the ICT-I's clients.

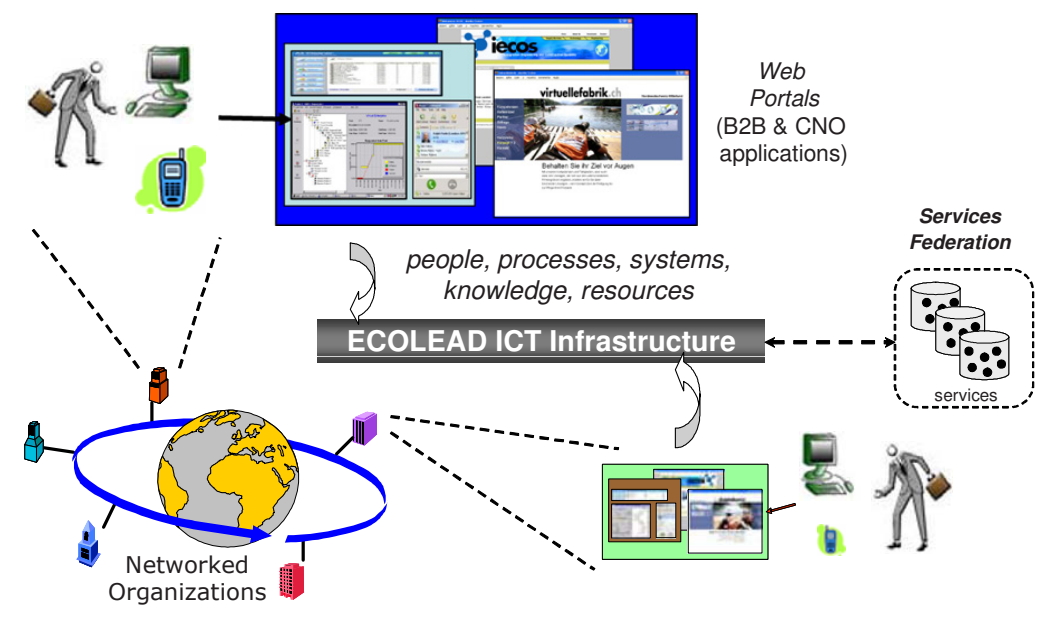

Figure 3 - General ICT-I usage scenario

\subsection{Interoperability}

Interoperability plays an essential role in any infrastructure where $\mathrm{CNO}$ actors and their applications are distributed and heterogeneous. In this context, Interoperability is seen as the ability of a system or a product to work with other systems or products without special effort from the customer or user'.

Interoperability is a very wide area, comprising since low-level sensors integration till higher levels of inter-organization collaboration. Regarding the core focus of ECOLEAD ICT-I, interoperability aspects are covered only at its essentials, i.e., interoperability issues are tackled by each ICT-I service according to its very specific needs, also benefiting from existing software and approaches.

An extremely important enabler for interoperability is the use of standards. Large international initiatives (e.g., OMG, OASIS, W3C and TeleManagement Forum) have been creating specifications with large acceptance by software developers and vendors worldwide. Therefore, to mitigate interoperability problems, the ECOLEAD ICT-I has been fully developed based on ICT standards, independent of computer platforms. Yet, all the current available ICT-I services have been formally specified independent of technology, using the UML methodology, meaning that they can be 
implemented in several languages and environments. ICT-I services have been implemented, however, as WS, which is a particular technology. On the other hand, WS is considered the standard de facto for implementing SOA-based systems.

Another relevant aspect to support the ICT-I vision is the possibility of invocation/interaction among WS deployed in different B2B frameworks. In this sense, WSIF standard ${ }^{10}$ is a strategic element. Thanks to it, collaboration can be much enlarged as any company can put available and share its services with CNO members seamlessly if services are prepared for that (Piazza and Rabelo, 2007).

\subsection{SaaS \& Utility Models}

In order to provide an affordable and 'made to fit' ICT-I for companies, ICT-I applies some principles of the SaaS-U (Software-as-a-Service / Utility) model ${ }^{11}$. In the SaaS model, software access is subscription-based, remotely hosted, and delivered over the Internet, without the need of complex implementations and IT infrastructure. This also means to allow on-demand services from large-scale and ubiquitous environments, where services can be accessed from everywhere and can be composed according to business rules and models. All this means that ICT-I doesn't require any local deployment. Its services are accessed remotely, upon request, paid-per-use, based on a contract (SLA - service license agreement) for hosting, managing and providing access to its services, no matter where the services providers are and how services have been deployed in the given WS frameworks.

\subsection{Services Federation}

A fundamental concept in the ECOLEAD ICT-I is the so-called Services Federation (see figure 3). This concept was adapted from Sun (Sun, 1999) to be used in the ICT-I environment, meaning that CNO-related services can belong to a virtual logical entity, the Services Federation. This federation comprises all services that can be reached, used and shared among CNO members, involving the ones related to: i) the ICT-I lifecycle; ii) the supporting services for high-level applications (i.e. the ICT-I itself); iii) the CNO life cycle (comprising VBE, PVC and VOM vertical services [see Chapter 4]); and iv) legacy / (intra-organization wrapped) systems services.

This is however transparent to users and applications. From the ICT-I point of view, services are invoked, searched, discovered and properly executed no matter which web services are needed to support a collaborative transaction, where they are, how they should be executed, and which technologies have been used in their implementations. Providers of such services can be both CNO members and independent software providers/vendors, having their own policies and rules to manage the services repositories. This means that ICT-I clients involve not only CNO client applications, but also CNO services providers.

ICT-I is evolving as the Services Federation is to be a dynamic and selfmanageable entity, with new members and services being incorporated to (or modified) and others being withdrawn from it. A given service may have different implementations available over the network. 


\subsection{Security Framework}

Security is a crucial issue in CNOs as several sensible information need to be accessed to guarantee the adequate management of CNOs. Organizations are often very skeptic to share information, and this is worse when there is a need to collaborate with unknown partners. Part of the problem is related to the lack of trust, both between organizations and in their systems. Therefore, security is a must to reinforce trust building and, as such, it should be managed properly.

Managing security is very complex. Considering that most of organizations in a CNO are composed of SMEs and that Virtual Organizations $(\mathrm{VO})^{12}$ are per definition a unique business, the security mechanisms to support the sharing and information access should be flexible and easily configurable, also considering the possible different laws each partner's country has. This aims at allowing a quick visibility setup of companies' information according to each VO needs and of the partners' roles in every VO. Ideally, this should be dynamically made and adjusted as long as business processes are executed and hence the information access by the involved partners can be controlled accordingly. Besides this functional complexity, other problems create tremendous difficulties for this envisaged scenario.

Aiming at filling this need, a security framework to cope with $\mathrm{CNO}$ requirements has been devised (Sowa et al., 2007). It supports AAA (Authorization, Authentication and Accounting) mechanisms and secure authenticated channels, allowing SMEs to configure the security levels and mechanisms for each VO they are involved in. The main element of the security framework is DRACO (Dynamic Responsibility Authorization for Collaborative Organizations) (Sowa et al., 2007).

The security framework is embedded in the ECOLEAD ICT-I. This prevents CNO users both from dealing with the usual complexity to deploy security systems locally, and from knowing security aspects in details. DRACO development has focused on handling CNO requirements. Other necessary security elements came from other reference international initiatives in the security area, namely WSFederation ${ }^{13}$, Liberty Alliance ${ }^{14}$ and WS-Trust ${ }^{15}$.

Another facet of the developed security framework is on how security is used by client / CNO applications. Instead of embedding security cares in the software code, DRACO uses a declarative approach to support security transparency. This means to move main security aspects to the server level, avoiding applications and web pages to have any code about security into them. This approach provides means for selecting the security functionality that is effectively required by a given application / service. Once a given service is chosen and VO roles are assigned to by the VO manager, the information access rights configuration is dynamically and automatically set up, and the required security mechanisms can be used.

\section{ICT-I SERVICES}

In order to provide an open and scalar model, ICT-I has a Reference Architecture (ICT-I-RA) from which instances-of it can be derived for different CNOs. In theory, it comprises "all" the possible classes of services than can be useful for any kind of CNO manifestation. This generalization has considered the three kinds of CNOs tackled in ECOLEAD: Virtual organization Breeding Environment (VBE), Virtual 
Organizations (VO) and Professional Virtual Communities (PVC). The ICT-I-RA goal is to be the base for a globally coherent derivation of particular CBIs for given CNOs; in this case, it was used to derive the ICT-I (Rabelo and Sesana, 2008).

\subsection{The ICT-I Reference Framework}

Figure 4 shows reference framework derived from ICT-I-RA. Actually, this represents one vision in terms of which concrete services could be derived from the ICT-I-RA's abstract classes. For instance, the abstract class "human collaboration services" is seen as a CSCW issue (for further implementation), which in turn has been thought as groupware, officeware and product development supporting services. It is up to the system designer and developer to implement his view about e.g. the groupware service (for example, including services like mailing, blog, videoconference, etc.). The other boxes represent the equivalent derivations from the other abstract classes.

Despite the performed derivation, only some of these services have been indeed implemented in ECOLEAD. Services in grey scale represent the developed services, whereas the black ones are supported only at a basic level. Services in white represent those that were not implemented but that can be further added to the framework in the future. Anyway, the implemented services support several of the most relevant requirements for $\mathrm{CNO}$ activities.

ICT-I services are positioned at different layers. Horizontal services are the ones independent of any specific type of CNO. For example, knowledge search service can be useful both to VBE and VO management. Basic services are domainindependent and are essentially used by other services - mainly by horizontal services - to support the complete and correct execution of a collaborative transaction. Platform Specific Services is a layer to cope with the fact that, in practice, services (both Basic and Horizontal) require specific tools and/or services when deployed. Therefore, they are intrinsically dependent on the services' implementation. Finally, Legacy system services are the ones that provide information about activities inside a given company to satisfy CNO needs. They use to be implemented in heterogeneous platforms and native front-ends, typically representing ERP systems and corporate databases. It shall be pointed out that legacy system services don't belong to ICT-I, but they may belong to the services federation.

In ECOLEAD, web-services technology and other associated standards (e.g. SOAP, UDDI and WSDL) have been chosen for implementation. Although this technology particularization, the specification of the services are made using UML methodology, which in turn makes the services specification independent of platform and, as such, they can be implemented using other technologies (e.g. webservices without SOAP or UDDI) and supporting tools (either open-source or COTS), depending on the envisaged CNO to support.

In resume, and considering the requirements stressed in chapter 2 and the features described in chapter 3, a CBI derived from the ECOLEAD ICT-I-RA - and hence that it was derived for the ECOLEAD project - is defined as an open, distributed, scalable, transparent and security-embedded collaborative serviceoriented infrastructure, tailored to support CNOs in the modeling and execution of collaborative tasks, accessed on-demand and paid-per-use. 

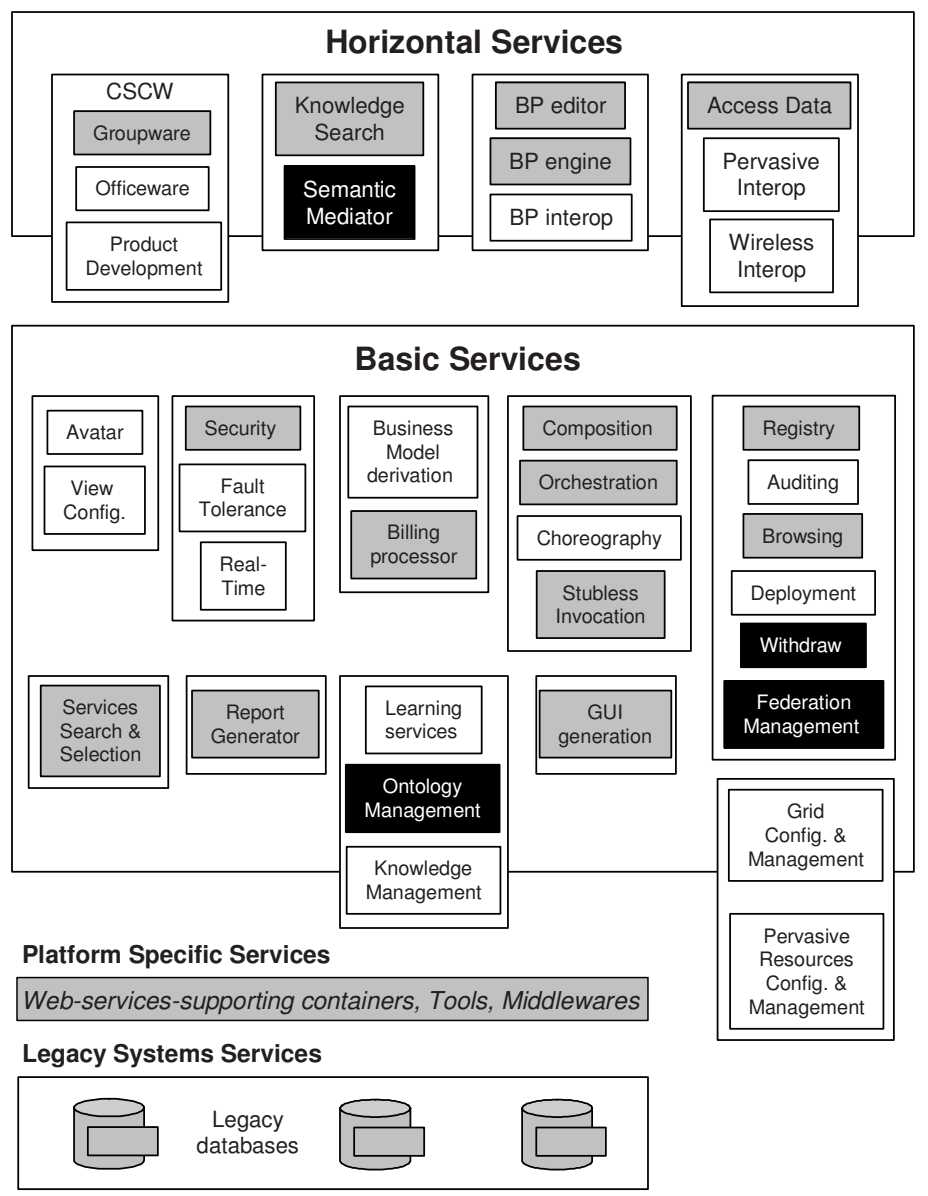

Figure 4 - ICT-I Reference Framework

\subsection{Implemented Services}

This section explains in very general terms the implemented services.

Horizontal Services

- CNO Actors On-Demand Collaboration Services. For supporting human collaboration: mailing, chat, task list, file storage, notification, calendar, wiki, forum, voice and syndication.

- CNO Knowledge Search Services. For supporting knowledge sharing, empowering the management of distributed and heterogeneous bodies of knowledge exposed by CNOs. Proper ontology and reconciliation rules are used for bridging some semantic gaps among knowledge repositories, allowing seamless retrieval of information.

- Interactive, user-centered BP Management Services. For supporting business process interconnection, on top of an existing open-source BPM environment (modeling module and execution engine), ICT-I provides support to task- 
oriented, interactive decisional activities to be performed by $\mathrm{CNO}$ actors. The forthcoming BPELAPEOPLE standard can also be used for that.

- CNO Data Access Services. For supporting systems interoperability, ICT-I offers services to support an easy and secure access to CNO members' databases, which includes a support for their definition and configuration and for the information that has to be shared.

\section{Basic Services}

- ICT-I Security Services. These services support confidentiality, integrity, availability and authentication in the communications. This includes the log-in and user management service.

- ICT-I Billing Services. They allow the implementation of different billing models to support the pay-per-use and on-demand service provision.

- ICT-I Services Composition. This service provides facilities to define and execute composed services, preferably using BPEL standard for services composition.

- ICT-I Reporting Services. For supporting the generation of reports to other services (e.g. "detailed billing usage", "services bill summary"), using predefined templates in well known formats (e.g. pdf, XML, HTML).

- ICT-I Services Registry and Discovery. For supporting the publishing of the web services in a UDDI repository as well as the search and browsing of services. These services also include the management of the ICT-I life cycle, involving services associated to its deployment, plugging, use, maintenance, unplugging and undeployment.

ICT-I services can be accessed via the ECOLEAD portal, from which users can have access to the ICT-I UDDI. This UDDI is so far centralized and it has a link to each individual service. Actually, so far this UDDI represents the Services Federation. As services have been developed by different partners, the UDDI makes the automatic link between the client application and the site where the invoked service is physically deployed.

The very detailed specification and implementation model of each implemented service is presented in Ratti and Rodrigo (2007). Some services are to be transparent to the users / clients, whereas other services are designed to assist end-users in some tasks. Figure 5 shows a graphical interface of an Editor of iBPM service (Ratti and Gusmeroli, 2007), which is one of the developed services. This editor allows the combination of human tasks and interactions with BPEL processes, creating a socalled collaborative business process. 


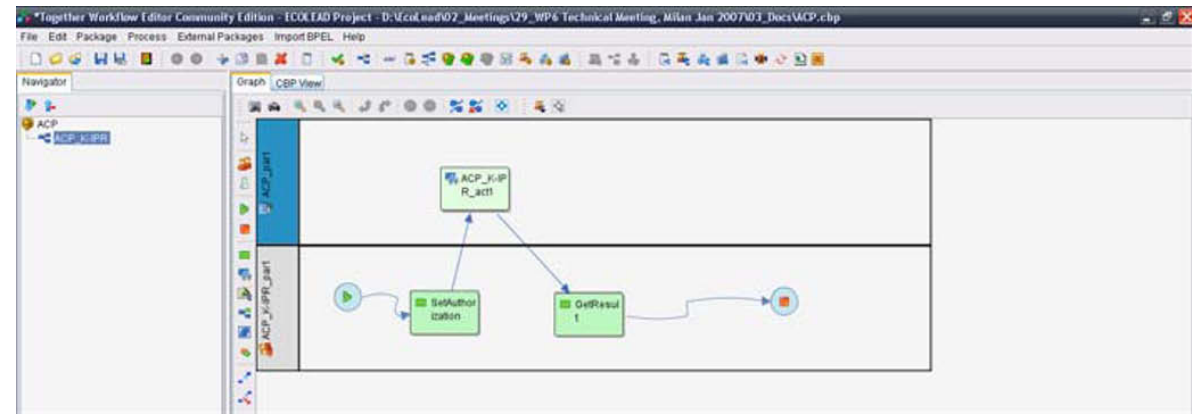

Figure 5 - iBPM editor

\section{CONCLUSIONS AND GENERAL ASSESSMENT}

This paper has presented the Collaborative Business ICT infrastructure (ICT-I) that was developed in the ECOLEAD Project for supporting CNOs in collaborating and doing businesses more effectively. It has been conceived based on the service oriented architecture paradigm / web-services technology, providing organizations with a transparent (mostly), platform-independent, easy-to-use, secure-embedded, lean, distributed, scalar, on-demand and pay-per-use ICT-I. Its conception and features are in line with some relevant initiatives, like NESSI ${ }^{16}$ and ATHENA ${ }^{8}$.

Supporting CNOs imply in a different set of functionalities, meaning that ICT-I complements B2B functionalities Thanks to the ICT-I framework flexibility and strong utilization of standards, all these functionalities can be integrated in the same computing environment (web portals, for instance) so that users do not need to see them as separate systems.

From the technological point of view and following current trends, ECOLEAD ICT-I has as main features:

- It is a web-based integrated platform devoted to CNOs, meaning that users should only have a browser and internet access. No local deployments;

- Services are accessed on demand This means having almost an 'a la carte' environment to fit each organization's needs.

- $\quad$ Services are paid per use, respecting variable and flexible business models;

- It is open and flexible to embrace new services without any interference in the use of the infrastructure, also meaning that ICT-I is scalable;

- As most of the ICT-I services are web-based and can be accessed via portals (portlets), they can also be accessed through mobile devices;

- Services \& data access are dynamically controlled by a flexible security system.

These features cope with requirements for an effective usage of this by SMEs. Actually, regarding its $\mathrm{CNO}$ orientation and other features stressed along the paper, it is believed that ICT-I is unique and it clearly goes towards supporting several requirements of Web 2.0 and Enterprise 2.0.

However, so far there is no support in terms of wider methodologies to guide a derivation process (e.g. using Model Driven Architecture approach), and to assist companies to overcome interoperability problems (e.g. ATHENA roadmap).

At the individual services and implementation level one can say that there are 
some equivalent services available in the Internet that offer equivalent functionalities. Yes, indeed. Actually, all the developed services made use of open source software available in a way to avoid the "re-invention of the wheel" and to add value on top of them when both integrating them in a common and relatively user-friendly (for an infrastructure) environment, and adapting them to some CNO requirements. Other more global issues are foreseen only at the conceptual and very generic level, such as fault tolerance mechanisms, services federation management, mobile access, etc. No support, or only very basic, is offered for issues like these.

Its validation has been so far achieved non-formally and partially. Non-formally because any supporting derivation methodology has been developed to guide the creation of particular business infrastructures for given CNOs. It is assumed that it is validated by the simple fact that a concrete business infrastructure (the ICT-I) was successfully implemented, i.e. it was possible to create a given instance of the ICT-I Reference Architecture following formal specifications of the derived services. Partially, because every single ICT-I service couldn't be exhaustively tested in many scenarios and real case situations. So far they were only tested in some trials and take-up-based scenarios. In terms of performance, level of transparency, plug \& play effectiveness, etc. of the ICT-I services, a detailed but preliminary analysis is provided in Ratti and Rodrigo (2007).

From the conceptual point of view, ICT-I is similar to what Salesforce.com's platform ${ }^{17}$ does. The difference is that this offers only CRM-related (web) services, which are physically centralized at the Salesforce company and whose services are provided by itself. Customers usually pay a fee monthly or pay according to the number of users a company has. ICT-I offers CNO supporting services independent of domain, and it is totally open and scalar, where providers can be any organization, including CNO members.

Web-service technology, despite its potentialities and increasing acceptance, has some drawbacks (e.g., it is stateless) that should be managed depending on the desired business process's behavior. Dealing with large-scale fault-tolerance platforms is still an open and very complex topic of research so it is expected that future outcomes of this can be incorporated in the ICT-I. Another complex issue is the management of the services federation. Each service provider can determine its own operational and security rules besides having different levels of computing infrastructures to run services, which can create serious troubles and to lead to other class of interoperability problems when several providers were established. Moreover, the operational policies should deal with the different life cycles of each service that is made available, which is also complex. Services should be easily discovered and immediately integrated/bound to workflow or orchestration systems mechanisms, but this bumps into the different ways and semantics the diverse providers have registered the services, on how the services interfaces (WSDL) are expressed, and if context awareness has to be considered.

All these difficulties represent challenges in the web-related community in spite of several ongoing works. Two interesting research projects that can be mentioned and that are dealing with some of those problems are DBE ${ }^{18}$ and ABILITIES ${ }^{19}$, but their results are still not at a level for being now used. These problems are essentially related to the technological perspective of the difficulties and impacts the adoption of the CNO paradigm by companies and the use of such kind of CBI to support their collaboration tends to provoke. Other perspectives, e.g. organizational, 
cultural, financial, among others are also extremely relevant and must be dealt with for the successful realization of the CNO paradigm. These perspectives are, however, out of scope of this paper.

\subsection{Acknowledgments}

This work was mostly supported by the European Commission under the project IST FP-6 IP ECOLEAD project (www.ecolead.org). The Brazilian participation was also supported by the Brazilian Council for Research and Scientific Development CNPq (www.cnpq.br) in the scope of IFM project (www.ifm.org.br).

\section{REFERENCES}

1. Camarinha-Matos, L. M.; Afsarmanesh, H.; Ollus, M.; 2005. Virtual Organizations: Systems and Practices, Springer.

2. Camarinha-Matos, L. M.; Afsarmanesh, H.; 2004 . Towards Next Business Models. In Collaborative Networked Organizations: a research agenda for emerging business models, Kluwer Academic Publishers, pp. 3-6.

3. Piazza, A.; Rabelo, R. J.; 2007. An Approach for Seamlessly Interoperation among heterogeneous web services-based B2B Frameworks [in Portuguese], Proceedings $8^{\text {th }}$ Brazilian Symposium on Intelligent Automation, pp. 451-458.

4. Rabelo, R.; Sesana, M.; 2008. Technical Report (Deliverable) D61.1d - ICT-I Reference Architecture and Framework for CNOs, in www.ecolead.org.

5. Ratti, R.; Rodrigo, M.; 2007. Technical Report (Deliverable) D64.1d - ICT-I Services, in www.ecolead.org.

6. Ratti, R.; Gusmeroli, S. (2007). Interactive User-Centered Business Process Management Services, in Proceedings PRO-VE'2007 - 8th IFIP Working Conference on Virtual Enterprises, in Establishing the Foundation of Collaborative Networks, Springer, pp. 487-494.

7. Sowa, G.; Sniezynski, T.; 2007. Technical Report (Deliverable) D64.1b - Configurable multi-level security architecture for CNOs, in www.ecolead.org.

8. SUN - JINI Technology Architectural Overview, http://www.sun.com/jini/whitepapers/ architecture.html, Jan 1999, in 30/01/2008.

\footnotetext{
${ }^{1}$ This is essentially based on the Companion Roadmap: www.companion-roadmap.org

${ }^{2}$ www-306.ibm.com/software/websphere/

${ }^{3}$ www.sap.com/platform/netweaver

${ }^{4}$ www.oracle.com/applications/fusion.html

${ }^{5}$ www.ibm.com/developerworks/lotus/products/notesdomino/

${ }^{6}$ sourceforge.net/projects/phpcollab/

${ }^{7}$ www.microsoft.com/biztalk/default.mspx

${ }^{8}$ ws.apache.org/axis/

${ }^{9}$ www.atena-ip.org

${ }^{10}$ ws.apache.org/wsif

${ }^{11} \mathrm{http}: / / \mathrm{www}-304 . \mathrm{ibm} . \mathrm{com} / \mathrm{jct} 09002 \mathrm{c} / \mathrm{isv} / \mathrm{marketing} / \mathrm{saa} / \mathrm{index} . \mathrm{html}$

${ }^{12}$ A Virtual Organization is a virtual and temporary grouping of organizations which is formed to attend to a given business opportunity.

${ }^{13}$ www.ibm.com/developerworks/library/specification/ws-fed

${ }^{14}$ www.projectliberty.org

${ }^{15}$ docs.oasis-open.org/ws-sx/ws-trust/v1.3/ws-trust.html

${ }^{16}$ NESSI Strategic Research Agenda - Framing the future of the Service Oriented Economy. Version 2006-2-13 (http://www.nessi-europe.com/documents/NESSI_SRA_VOL_1_20060213.pdf); ICT for Enterprise Networking (http://cordis.europa.eu/ist/directorate_d/en_intro.htm).

${ }^{17}$ www.salesforce.com

${ }^{18}$ www.digital-ecosystem.org

${ }^{19}$ services.txt.it/abilities
} 